\title{
Falta às consultas médicas agendadas: percepções dos usuários acompanhados pela Estratégia Saúde da Famillia, Manguinhos, Rio de Janeiro
}

\author{
Absence to scheduled medical appointments: perceptions of the users of the Family Health Strategy, Manguinhos, \\ Rio de Janeiro
}

\section{La ausencia de las citas médicas programadas: percepciones de los usuarios acompañados por la Estrategia Salud de la Familia, Manguinhos, Rio de Janeiro}

Mellina Marques Vieira Izecksohn. Centro de Saúde Escola Germano Sinval Faria (CSEGSF). Rio de Janeiro, RJ, Brasil. mellizeck@gmail.com (Autora correspondente)

Jaqueline Teresinha Ferreira. Universidade Federal do Rio de Janeiro (UFRJ). Rio de Janeiro, RJ, Brasil. jaquetf@gmail.com

\section{Resumo}

Introdução: os motivos que levam o usuário da Estratégia Saúde da Família a comparecer ou não às consultas médicas programadas precisam ser conhecidos devido ao impacto negativo que as faltas podem causar no serviço de saúde, inclusive de caráter financeiro e no cuidado do usuário. Objetivo: conhecer o motivo e a percepção do usuário quanto à importância de comparecer às consultas agendadas, identificando as razões para 0 não comparecimento. Métodos: pesquisa quanti-qualitativa, com quantificação de faltosos em duas equipes do Centro de Saúde Escola no período de seis meses e entrevista semiestruturada com 22 pacientes que faltaram ou não às consultas médicas agendadas. Resultados: foi identificado um percentual de faltas de 48,9\% no período estudado. 0 principal motivo para agendamento das consultas dos usuários foi 0 acompanhamento de sua saúde, 0 que pode ter diversas interpretações por parte dos profissionais de saúde e dos pacientes. Entre os motivos citados para as faltas às consultas, destacaram-se 0 esquecimento assim como 0 agendamento em horários inoportunos. Alguns ruídos na comunicação entre os usuários e a unidade de saúde também foram identificados, como a impossibilidade de cancelamento do encontro sem que o usuário compareça ao serviço. Conclusões: 0 estudo permitiu a identificação de aspectos relacionados à organização do serviço de saúde e ao usuário que interferem na assiduidade, assim como das propostas que podem melhorar o comparecimento às consultas médicas, tais como diversificar as formas de agendamento, contato telefônico prévio, escutar o usuário, entre outros.

\section{Abstract}

Introduction: the reasons that lead users of the Family Health Strategy (FHS) to attend or not scheduled medical appointments must be known due to the negative impact that non-attendance can cause in the health service, including financial consequences and those related to the quality of care. Objective: to know the reasons for non-attendance and the users' perception of the importance of attending scheduled appointments, identifying the reasons for non-attendance. Methods: quantitative and qualitative research, identifying non-attenders in two FHS teams of an Academic Health Center, within a six months period, and conducting semi-structured interviews with 22 patients who missed or attended scheduled medical appointments. Results: the study found a non-attendance rate of $48.9 \%$ during the study period. The main reason for scheduling an appointment was to monitor one's health, which can have various interpretations on the part of health professionals and patients. Among the reasons for non-attendance were forgetting the appointment and the fact that the appointment was scheduled for an inconvenient time. Some communication problems between users and the Health Unit were also identified, such as the impossibility of canceling the appointment without having to go to the health unit. Conclusions: the study identified aspects related to the organization of the health service and to the users that interfere with attendance, and also made it possible to propose measures to improve attendance, such as offering different ways for scheduling appointments, prior telephone contact and listening to the user.

Palavras-chave:

Agendamento de Consultas Absenteísmo

Saúde da Família Assistência Ambulatorial

Keywords:

Appointments and Schedules Absenteeism Family Health Ambulatory Care

\section{Fonte de financiamento: declaram não haver. Parecer CEP: ENSP/FIOCRUZ n. 195.406 \\ Conflito de interesses: declaram não haver. \\ Recebido em: 25/06/2014. Aprovado em: 19/08/2014.}




\section{Resumen}

Introducción: las razones que llevan al usuario de la Estrategia Salud de la Familia para asistir o no a las citas médicas programadas deben ser conocidas por el impacto negativo que causan fallas en el servicio de salud, incluidas las disposiciones financieras y de atención al usuario. Objetivo: conocer la percepción de los usuarios sobre la importancia de asistir a citas programadas, y la identificación de las causas de la ausencia. Métodos: investigación cuantitativa y cualitativa, con la cuantificación de ausentes en dos equipos de Centro Médico Académico en uno semestre, y entrevistas semi-estructuradas con 22 pacientes que tengan asistido o no a las citas programadas. Resultados: fue identificado un porcentaje de ausencias de 48,9\% durante el período de estudio. La razón principal de la programación de las consultas fue el seguimiento del estado de salud que puede tener diversas interpretaciones por parte de los profesionales sanitarios y de los pacientes. Entre las razones de citas perdidas pararón el olvido y la programación del horario en momentos inoportunos. También se han detectado algo de ruido en la comunicación de los usuarios con la Unidad de Salud, como la imposibilidad de cancelar la cita sin tener que volver allá. Conclusiones: el estudio permitió la identificación de los aspectos relacionados con la organización del servicio de salud y el usuario que interfieren con la asiduidad, e identificar propuestas que puedan mejorar la asistencia a las citas médicas, como formas de diversificar las formas de programación, el contacto telefónico previo, entre otros.

\section{Introdução}

Os motivos que levam o usuário da Estratégia Saúde da Família (ESF) a comparecer ou não às consultas médicas programadas precisam ser conhecidos, devido ao impacto negativo que as faltas podem causar no serviço de saúde, seja financeiramente, no processo de trabalho ou no cuidado ao usuário. ${ }^{1,2}$ Os meios de comunicação, como jornais impressos e digitais, têm se voltado para este problema e chamam a atenção para o fato do absenteísmo às consultas dificultar o acesso ao serviço de saúde, com aumento das filas de espera para agendamento. ${ }^{3,4,5,6}$ Além disso, o usuário faltoso tende a agendar uma nova consulta, comprometendo mais um horário de atendimento que poderia ser destinado a outra pessoa, o que pode, nesses casos, dar uma falsa impressão de falta de profissional ou de vagas causada pelo mau aproveitamento do serviço ofertado.

Poucos são os estudos brasileiros que abordam esse tema, principalmente se nos ativermos à percepção do usuário. Os estudos existentes nacionais e internacionais que buscam analisar as razóes das faltas às consultas referem como fatores determinantes a distância entre a data do agendamento e a consulta, esquecimento, dificuldade de liberação do trabalho e de locomoção, paternidade, melhora do sintoma que ocasionou o agendamento e atendimento médico prévio sem que seja agendado, além de relatos do usuário não ter sido avisado da marcação. . $7,8,9,10,11,12,13^{2}$

A literatura aponta igualmente alternativas que já foram pensadas para diminuir as faltas às consultas: aviso pelo correio, marcação individualizada considerando-se o perfil do usuário; não remarcação automática das pessoas que faltam às consultas; adaptação da marcação ao horário de trabalho do usuário; lembrete do agendamento no dia anterior pelo agente comunitário de saúde (ACS); entre outras. ${ }^{78,9,14}$ No entanto, em algumas situaçóes, os resultados não são expressivos, o que reforça a noção de que, sem se entender a percepção do usuário, torna-se difícil reduzir esse problema.

O objetivo deste artigo é conhecer o motivo e a percepção do usuário quanto à importância de comparecer às consultas agendadas, identificando as razôes para o não comparecimento à consulta em duas equipes de Saúde da Família do Centro de Saúde Escola Manguinhos.

\section{Métodos}

Este é um estudo descritivo-exploratório com abordagem quanti-qualitativa, tomando como base as reflexóes oriundas da dissertação apresentada como defesa do Mestrado Profissional em Atenção Primária à Saúde, da Escola Nacional de Saúde Pública Sérgio Arouca (ENSP) da Fundação Oswaldo Cruz (Fiocruz), em setembro de 2013.

Após levantamento bibliográfico realizado no período de quatro meses, realizamos a quantificação do número de faltas dos usuários às consultas médicas de duas Equipes de Saúde da Família do Centro de Saúde Escola Manguinhos, atualmente incorporado ao Centro de Saúde Escola Germano Sinval Faria, no período de julho a dezembro de 2012. Tais dados foram obtidos por levantamento do banco de dados do prontuário eletrônico ALERT, utilizado como fonte de informação da unidade desde abril de 2008. 
A seguir, realizamos entrevistas semi-estruturadas, pautadas em um questionário com perguntas abertas e fechadas, com usuários das mesmas duas equipes, com o objetivo de compreender como é realizado o agendamento e o porquê de eles terem comparecido ou não às consultas agendadas. As entrevistas foram realizadas com onze indivíduos que compareceram e onze que não compareceram às consultas agendadas. $\mathrm{O}$ trabalho de campo foi organizado mediante agendamento prévio de visitas domiciliares da pesquisadora com os agentes comunitários de saúde e as famílias, sendo as entrevistas realizadas no domicílio dos usuários.

$\mathrm{Na}$ entrevista, abordamos as representaçóes da importância das consultas agendadas e o motivo para o não comparecimento na perspectiva dos usuários. As entrevistas foram gravadas e realizadas após o consentimento do entrevistado, com duração média de 25 minutos. Excluímos da amostra gestantes e usuários menores de 18 anos por levantarem questóes relacionadas ao cuidado do outro que não foram abordadas neste estudo.

As duas equipes utilizam apenas o sistema eletrônico para agendamento das consultas médicas. As unidades funcionam de segunda à sexta-feira, no horário de 08 às 17 horas, e a marcação pode ser feita durante todo o expediente. Até o momento, não há outra forma que não seja a presencial para realizar o agendamento.

Como as entrevistas foram realizadas no período de funcionamento da unidade, diminuiu-se a chance de se entrevistarem usuários que estivessem trabalhando fora da residência nesse período, sendo essa uma limitação do estudo. Alguns, por trabalharem em dias alternados, tiveram as entrevistas agendadas para quando estivessem em casa.

O trabalho foi inicialmente apresentado às equipes escolhidas durante reunião das mesmas, mediante autorização prévia dos médicos e enfermeiros, tendo aceitação, aprovação e colaboração por parte das mesmas, o que facilitou a coleta de dados.

A amostra foi intencional sendo limitada por saturação ou redundância de informaçóes, segundo Fontanella, ${ }^{15}$ chegando ao número de 22 entrevistados.

A interpretação das respostas buscou significados a partir da construção de categorias temáticas, ${ }^{16}$ visando à sua articulação em conjuntos mais amplos, que se relacionavam com conceitos representativos das ciências sociais e humanas em saúde. Assim, os temas foram selecionados após leitura exaustiva e análise das entrevistas, o que proporcionou a escolha de categorias mais evidentes e significativas presentes nos textos, os quais também foram confrontados com a literatura científica. Dessa forma, a análise temática permitiu descobrir os núcleos de sentido que compuseram esta comunicaçáo.

\section{Cenário e universo de estudo}

O cenário do estudo constitui-se no complexo de Manguinhos, situado na Zona Norte da cidade do Rio de Janeiro, com 36.160 moradores ${ }^{17}$ renda per capita de $\mathrm{R} \$ 118,8624,{ }^{17} \mathrm{IDH}$ de $0,72624,{ }^{17}$ encontrando-se na $122^{\text {a }}$ colocação em relação ao $\mathrm{IDH}^{17}$ entre os 126 bairros do município.

As duas equipes estudadas estáo completas, ${ }^{18}$ compostas pelo mesmo médico, enfermeiro, técnico de enfermagem e agentes comunitários de saúde há mais de um ano, sendo que ambas recebem alunos de graduaçáo de medicina da Universidade Federal do Rio de Janeiro e uma delas ainda recebe um médico residente de Medicina de Família e Comunidade da ENSP. Tais equipes foram escolhidas por essas razóes e igualmente por serem equipes distintas daquela da qual a pesquisadora faz parte, proporcionando uma maior relativização para a realização deste estudo. Foram garantidos o sigilo e a privacidade dos sujeitos e das equipes em relação aos dados confidenciais e, para isso, utilizamos nomes fictícios para os sujeitos da pesquisa, identificados por letras, bem como para as equipes, nomeadas como equipe A e equipe B.

A população abrangida pela equipe A é de 2743 pessoas, das quais 14 são gestantes e 811 têm menos de 18 anos, sendo a amostra de estudo composta por 1918 pessoas. A equipe B abrange uma comunidade com 2624 usuários, sendo 681 menores de 18 anos e 17 gestantes, sendo a amostra de estudo 1926 pessoas.

$\mathrm{O}$ agendamento das consultas é realizado preferencialmente na unidade, pelo usuário, ao se dirigir à baia da equipe na sala de espera do centro de saúde, sendo o mesmo realizado pelo agente comunitário ou por qualquer outro profissional da equipe de saúde presente, a saber: técnico de enfermagem, enfermeiro ou médico.

O estudo foi aprovado pelo Comitê de Ética em Pesquisa da Escola Nacional de Saúde Pública Sérgio Arouca (ENSP), sob o número 195.406, em 07 de fevereiro de 2013. 


\section{Resultados e Discussão}

No período estudado de seis meses, foi identificado um percentual de faltas de 48,9\% do total de 2272 agendamentos, sendo que, dessas faltas, $58,5 \%$ das pessoas faltaram uma vez, 26,5\% faltaram duas vezes e $15 \%$ faltaram mais de três vezes. Do total de usuários agendados, entrevistamos 22 pessoas, das quais metade faltou à consulta e a outra metade compareceu.

Como as entrevistas foram realizadas durante a semana em período diurno, sabíamos que podíamos encontrar mais aposentados e donas de casa no total de entrevistados, assim quatro (18\%) eram aposentados por idade, tempo de serviço ou invalidez e seis $(27 \%)$ eram donas de casa.

Quanto ao gênero, 20 eram do sexo feminino e apenas 2 do sexo masculino. Quanto à faixa etária entrevistada, 13 pessoas tinham de 20 a 59 anos e nove eram maiores de 60 anos. Um destaque é que $64 \%$ dos assíduos são maiores de 60 anos e $82 \%$ dos faltosos possuem menos de 60 anos.

A renda individual encontrada foi baixa, sendo em sua maioria de 1 a 2 salários mínimos e a maioria dos entrevistados possui ensino fundamental incompleto.

O fato de a maioria dos entrevistados ser do sexo feminino pode ser justificado em função de as mulheres ainda serem mais fáceis de serem encontradas nos domicílios durante o dia. A maior proporção de entrevistados faltosos encontrados tem idade classificada como populaçáo economicamente ativa, o que concorda com alguns estudos que identificam que, com o aumento da idade, a proporção de faltas diminui. ${ }^{10,19}$ Esse fato pode estar associado à maior probabilidade de doença em faixas etárias mais altas, ou ao acúmulo de experiência, que leva o usuário a recorrer a uma consulta por identificar antecipadamente sinais de problemas relacionados à saúde. ${ }^{10,19,20}$

Quanto à escolaridade e à renda dos entrevistados, nenhum desses aspectos parece interferir na assiduidade às consultas médicas agendadas. No entanto, vale a reflexão de que todos os entrevistados possuem baixa renda e pouca escolaridade, não apresentando uma diferença significativa entre eles, além do número avaliado ser pequeno para uma análise quantitativa desses fatores.

Abordamos o usuário questionando sobre se lhe foi oferecida a oportunidade de opinar sobre o horário de atendimento. Quase todos os entrevistados afirmaram não terem sido ouvidos sobre o melhor dia ou período para agendamento, sendo desconsiderados, inclusive, seus horários de trabalho.

Isto pode ser evidenciado quando questionamos a Sra. D. sobre se tinha sido consultada a respeito do melhor horário para agendamento: “... não, ela marcou e pronto...", o mesmo foi evidenciado pelo Sr. P., que respondeu: "Não, não, se eles me dessem eu gostaria, mas náo dá não.".

Ao questionarmos os usuários se precisariam fazer alguma modificação de sua rotina diária para comparecer às consultas, a maior parte referiu necessitar de algum nível de rearranjo familiar, doméstico ou profissional. Como exemplo, temos usuários que mencionaram trabalhar em esquema de plantáo, em dias alternados, devendo marcar a consulta nos seus dias de folga, além de uma senhora que vende quentinhas, que teria facilitado seu comparecimento se fosse agendada para o período da tarde, já que passa a manhã cozinhando.

Outro dado que chamou a atenção foi a não participação dos usuários no agendamento. Alguns receberam em casa o aviso da marcação, e terminaram sendo marcados para dias em que estariam, por exemplo, ausentes do município, inviabilizando, portanto, seu comparecimento à consulta.

Observamos que, no entanto, nos casos em que os ACS possuem relaçôes próximas com os usuários, este cuidado pôde ser identificado, como relata a Sra. Y.: "O que ela (ACS) puder fazer por mim, ela faz, porque eu trabalho fora. Eu trabalho como plantonista, então ela tenta marcar nas minhas folgas." De qualquer forma, devemos preconizar que o tratamento seja cauteloso com todos os usuários e não somente com alguns.

Quando questionamos sobre o motivo dos entrevistados comparecerem às consultas, apenas um usuário mencionou a consciência de não estar tirando a vaga de alguém que precise, sendo que a maioria aponta a consulta marcada como um compromisso ao qual náo se deve faltar.

Quando nos referimos ao motivo de agendamento, este foi diversificado entre assíduos e faltosos. ${ }^{20}$ Para os assíduos, o "acompanhamento" de doenças foi o predominante, o que implica em receber o resultado ou solicitar exames e/ou medicação. ${ }^{21}$ As motivaçôes para agendamento variaram muito para os faltosos, aparecendo a questão do "acompanhamento", mas também queixas como queixas ginecológicas, álgicas ou dermatológicas. Duas pessoas desconheciam o motivo do agendamento. A 
dor é destacada em vários estudos como o principal motivo para a procura de atendimento médico, programado ou não, o que vai de encontro aos nossos achados. ${ }^{20}$

Nesse contexto, um estudo mostra que as pessoas faltam mais quando devem receber os resultados dos exames do que quando o objetivo é solicitá-los. ${ }^{20,21}$ Isso nos faz refletir que a realização do exame em si é percebida como uma atitude de cuidado pelos usuários.

A organização das duas equipes facilita o agendamento das consultas, com curtos períodos de espera entre a data da marcação e a realização da consulta e facilidade de reagendamento. O intervalo entre o dia do agendamento e a data da consulta foi considerado satisfatório pelos entrevistados e variou de um a 60 dias.

Diferentemente do relatado em algumas pesquisas, não foi encontrada relação direta entre o intervalo entre a marcação e a consulta com a assiduidade, sendo encontrado, inclusive, um tempo de espera menor dos faltosos que dos assíduos. ${ }^{11,22}$

Ao questionarmos sobre o reagendamento por parte do usuário - se seria realizado e por qual o motivo - apenas uma pessoa disse que não remarcaria a consulta por fazer "acompanhamento" em outro serviço.

A facilidade de reagendamento das consultas médicas nas duas equipes é vista por alguns usuários como um dos motivos de falta, sendo este evidenciado em algumas falas, como a da Sra. Y: "Já remarquei, não tive dificuldade, ele mesmo remarcou na hora." E do Sr X: "É fácil remarcar a consulta, a ACS vem na minha casa". Esta facilidade fica evidenciada pela alta frequência de pessoas que faltaram repetidas vezes à consulta médica agendada no período de seis meses, sendo que alguns usuários chegaram a faltar sete vezes. Alguns estudos mostram que as pessoas que faltaram às consultas foram atendidas nos três meses que se seguiram à data que não compareceram. Assim, o fim do reagendamento automático é mencionado como medida para reduzir o problema do absenteísmo. ${ }^{11}$

Um aspecto que se destacou como causa do absenteísmo e que apareceu insistentemente nas entrevistas foi o esquecimento. Alguns enfatizam que a "preguiça" e o "descaso" com sua própria saúde seriam motivos importantes nessa situação.

Esses dados nos trazem dois questionamentos: se aquela consulta era importante para esse usuário, ou seja, se ele valoriza esse encontro e o considera importante, ou se era demanda do serviço; e, ainda, qual a real necessidade dessa consulta para ele e para o serviço. Isso nos remete à questấo dos protocolos clínicos que desconsideram o indivíduo, onde este passa a assumir a identidade da doença que possui. Nesse contexto, as necessidades de saúde são interpretadas como o consumo de serviço, deixando de lado toda a sua complexidade e diferentes aspectos que a compóem. Assim, como as necessidades são individuais, a proposiçẫo de protocolos assistenciais por vezes não dá conta da especificidade da pessoa. ${ }^{23}$

Nessa unidade, o contato prévio com o usuário, por meio do envio de mensagem de texto para o celular, ajuda o usuário a lembrar do seu compromisso e é, possivelmente, uma ferramenta importante na redução do esquecimento, fato demonstrado em alguns estudos. ${ }^{8,9,14}$

No entanto, um problema identificado foi que nem todos os cadastros dos usuários possuem os números dos telefones. Além disso, no cadastro das crianças não consta o contato do responsável. Assim, um estudo de intervenção que identifique a redução ou não do número de faltas daqueles lembrados por meio de mensagem de texto pode ser pensado, sem esquecer da importância da atualização constante do cadastro dos usuários.

O trabalho como justificativa para falta às consultas surge em outros estudos, mas não com muito destaque, diferentemente deste em que ficou evidenciado por parte dos entrevistados quando abordamos a organização pessoal. ${ }^{8,9,10,11,12}$

Sra. K., quando questionada quanto ao motivo que leva os usuários a faltarem às consultas, mencionou que muitas pessoas priorizam o trabalho em detrimento da saúde: "As pessoas esquecem, vão trabalhar, ficam com isso na cabeça, trabalho, trabalho, trabalho, o tempo todo." Do mesmo modo, a Sra. X. explica que algumas pessoas receiam perder o emprego já que a falta, mesmo que justificada, é mal vista pelo empregador: "As pessoas que trabalham faltam por medo de perder o emprego".

Muitos usuários afirmam que seu empregador não aceita atestado ou o patrão vê com desconfiança aquele que vai às consultas no horário do expediente. Dessa forma, dependendo do seu horário de trabalho, do horário de funcionamento da unidade e da organização dos turnos pela equipe, não existe muita alternativa para os usuários comparecerem às consultas.

Assim, o conhecimento do cotidiano ${ }^{24,25}$ do usuário no momento do agendamento mostra-se importante para evitar marcaçôes em horários inoportunos que culminarão com a falta à consulta por questôes do trabalho ou mesmo familiares. 
Um estudo na Arábia Saudita, o único encontrado que especifica esse fator, mostra um maior percentual de faltas no período da manhã que nos atendimentos noturnos. ${ }^{21}$ Deste modo, um estudo que avalie o período em que as pessoas mais faltam às consultas pode ser pensado, inclusive comparando uma unidade que funcione em horário tradicional, de 8 às 17 horas, com uma que funcione em horário estendido, de 8 às 20 horas. ${ }^{26}$

Percebemos então que agendas fechadas, com turnos predeterminados por ciclo de vida ou comorbidades, ou unidades que náo trabalham com horário estendido tendem a gerar menor variedade de oferta para o usuário, que precisa se adequar àquele formato. Dessa forma, há um aumento do absenteísmo.

\section{Conclusão}

Ao buscar a visão do usuário, foi possível observar alguns aspectos que se destacaram, de modo que a perspectiva do usuário sobre o serviço mostrou-se deveras importante para compreendermos as suas faltas.

Assim, algumas mudanças no serviço podem ser benéficas para reduzir o absenteísmo, tais como: facilitar a desmarcação e o agendamento; ampliar as formas de comunicação da população com a unidade por meio de telefones e correio eletrônico; e ouvir o usuário e marcar em horário conveniente para ele, entendendo seu cotidiano, inclusive o que precisa organizar para comparecer à consulta.

Nesse estudo, iniciamos a discussão sobre a importância de se conhecer a percepçáo do usuário. Daqui para frente, muito ainda deve ser desvendado, principalmente no que tange à visão do profissional de saúde sobre o usuário, à visão do usuário sobre o serviço, se eles estáo se comunicando efetivamente ou se há apenas um diálogo vazio.

\section{Referências}

1. Ferreira J. A saúde em comprimidos: influências socioculturais na interpretação de sintomas e terapias medicamentosas em uma vila de classe popular de Porto Alegre. Saúde Debate. 2001;25(59):67-72.

2. Husain-Gambles M, Neal RD, Dempsey O, Lawlor DA, Hodgson J. Missed appointments in primary care: questionnaire and focus group study of health professionals. Br J Gen Pract. 2004;54(499):108-13.

3. Portal da Cidadania [Internet]. Excesso de faltas em exames e consultas prejudica a saúde de Biguaçu. Santa Catarina: Prefeitura Municipal de Biguaçu; 2011[acesso em 2011 Dec 05]. Disponível em:

http://www.bigua.sc.gov.br/excesso-de-faltas-em-exames-e-consultas-prejudica-a-saude-de-biguacu/.

4. Correio da Manhã [Internet]. Doentes faltam mais às consultas e exames. Portugal, Lisboa; 2012 [acesso em 2012 Sep 05]. Disponível em: http://www.cmjornal.xl.pt/detalhe/noticias/nacional/atualidade/doentes-faltam-mais-as-consultas-e-exames.

5. A Gazeta [Internet]. Faltas em consultas médicas chegam a 70\% na rede pública. Espírito Santo; 2011 [acesso em 2011 Dec 06]. Disponível em: http://gazetaonline.globo.com/_conteudo/2011/05/noticias/a_gazeta/dia_a_dia/847876-faltas-em-consultas-medicas-chegam-a-70-na-rede-publica.html.

6. G1 [Internet]. Falta às consultas marcadas gera prejuízo de R $\$ 500$ mil em Canoas, RS. Rio Grande do Sul; 2012 [acesso em 2012 Aug 04]. Disponível em:

http://g1.globo.com/rs/rio-grande-do-sul/noticia/2012/06/falta-consultas-marcadas-gera-prejuizo-de-r-500-mil-em-canoas-rs.html.

7. Kwintner M. When absence speaks louder than words: an object relational perspective on no-show appointments. Clin Soc Work J. 2011;39:253-61. http://dx.doi.org/10.1007/s10615-011-0313-x.

8. Giacchero KG, Miasso Al. Ambulatório de psiquiatria em hospital geral: caracterização da adesão de usuários ao agendamento. Rev RENE. 2008 [acesso em 2012 Aug 04];9(2):20-7. http://www.revistarene.ufc.br/revista/index.php/revista/article/view/547.

9. Perron NJ, Dao MD, Kossovsky MP, Miserez V, Chuard C, Calmy A et al. Reduction of missed appointments at an urban primary care clinic: randomized controlled study. BMC Fam Pract. 2010;11:79. http://dx.doi.org/10.1186/1471-2296-11-79.

10. Barron WM. Failed appointments: Who misses them, why they are missed, and what can be done. Prim Care. 1980;7(4):563-74.

11. Van der Meer G, Loock JW. Why patients miss follow-up appointments: a prospective control-matched study. East Afr J public Health. 2008;5(3):154-6.

12. Neal RD, Hussain-Gambles M, Allgar VL, Lawlor DA, Dempsey O. Reasons for and consequences of missed appointments in general practice in the UK: questionnaire survey and prospective review of médical records. BMC Fam Pract. 2005;6:47. http://dx.doi.org/10.1186/1471-2296-6-47.

13. Lacy NL, Paulman A, Reuter MD, Lovejoy B. Why we don't come: patient perceptions on no-shows. Ann Fam Med. 2004;2:541-5. http://dx.doi.org/10.1370/afm.123

14. Gerson LW, McCord G, Wiggins SL. A strategy to increase appointment keeping in a pediatric clinic. J Comm Health. 1986;11(2):111-21. http://dx.doi.org/10.1007/BF01321512. 
15. Ministério da Saúde (BR), Secretaria de Atenção à Saúde, Departamento de Atenção Básica. Política Nacional de Atenção Básica. Brasília: MS; 2012.

16. Weingarten N, Meyer DL, Schneid JA. Failed appointments in residency practices: who misses them and what providers are most affected? $\mathrm{J}$ Am Board Fam Pract. 1997;10(6):407-11.

17. Nour El-Din MM, Al-Shakhs FN, Al-Oudah SS. Missed appointments at a university hospital in eastern Saudi Arabia: magnitude and association factors. J Egypt Public Health Assoc. 2008;83(5-6):415-33.

18. Instituto Brasileiro de Geografia e Estatística. Censo Demográfico 2010 [Internet]. Rio de Janeiro: IBGE; 2010 [acesso em 2012 Jul 04]. Disponível em: http://censo2010.ibge.gov.br/.

19. Assis MMA, Villa TCS, Nascimento MAA. Acesso aos serviços de saúde: uma possibilidade a ser construída na prática. Ciênc Saúde Coletiva. 2003;8(3):815-23. http://dx.doi.org/10.1590/S1413-81232003000300016.

20. Lora AP. Acessibilidade aos serviços de saúde estudo sobre o tema no enfoque da saúde da família no município de Pedreira SP [dissertação]. São Paulo: UNICAMP; 2004.

21. Ferreira J. Semiologia do Corpo. In: Leal OF, editors. Corpo e Significado - ensaios de antropologia social. Porto Alegre: UFRGS; 1995. p. 89-104.

22. Fontanella BJB, Ricas J, Turato RB. Amostragem por saturação em pesquisas qualitativas em saúde: contribuições teóricas. Cad Saúde Pública. 2008;24(1):17-27. http://dx.doi.org/10.1590/S0102-311X2008000100003.

23. Souza CR, Botazzo C. Construção social da demanda em saúde. Physis (Rio J). 2013;23(2):393-413. http://dx.doi.org/10.1590/S0103-73312013000200005.

24. Mourão P R. Tempo decorrido desde a última consulta: análise de um modelo estatístico aplicado ao caso das mulheres na Espanha. AMB Rev Assoc Med Bras. 2011;57(2):164-70.

25. Whittle J, Schectman G, Lu N, Baar B, Mayo-Smith MF. Relationship of scheduling interval to missed and cancelled clinic appointments. J Amb Care Manage. 2008;31(4):290-302. http://dx.doi.org/10.1097/01.JAC.0000336549.60298.1d.

26. Secretaria Municipal de Saúde do Rio de Janeiro. Carteira de serviços - relação de serviços prestados na Atenção Primária. Rio de Janeiro [acesso em 2013 Jun 20]. Disponível em http://subpav.org/index.php?p=cart. 